\title{
Initial Experience With a Bioresorbable Polymer Anchor
}

\author{
Robert R. Burnham Jr. ${ }^{1}$, Jayanth Kumar ${ }^{2}$, Michael Pinzur ${ }^{1}$, Adam Schiff ${ }^{1}$ \\ 1. Orthopaedic Surgery, Loyola University Medical Center, Maywood, USA 2. Orthopaedic Surgery, Loyola University \\ Chicago Stritch School of Medicine, Maywood, USA
}

Corresponding author: Robert R. Burnham Jr., robert.burnham@lumc.edu

\section{Abstract \\ Background}

Anchors are frequently used in reconstructive orthopedic surgery to achieve fixation of soft tissue to bone. Anchors vary with respect to material composition, configuration, and methods of fixation at the site of attachment. The fixation component of anchoring devices has generally evolved from metal-fabricated implants to various types of bioresorbable anchors. The SonicAnchor ${ }^{\mathrm{TM}}$ (Stryker, Kalamazoo, MI USA) polymer implant provides a unique form of anchor fixation using SonicFusion ${ }^{\mathrm{TM}}$ technology to achieve interdigitation within cancellous bone while being radiolucent and providing a small footprint.

\section{Methods}

During a four-year period, 116 patients underwent a reconstructive orthopedic foot and ankle surgical procedure with the use of at least one bioresorbable polymer anchor (SonicAnchor implant). There were 59 males and 57 females, with an average age of 42 years (range: $12-83$ years).

\section{Results}

A total of 233 bioresorbable anchor (SonicAnchor) implants were used in 116 patients. Of the 116 patients, 108 (93.1\%) achieved successful clinical healing of their surgery at their most recent follow-up. The average follow-up duration was 309 days (range: 14-1,429 days). Eight (6.9\%) patients were lost to follow-up prior to clinical healing. Two (1.7\%) patients underwent reoperation. Also, $65(56 \%)$ patients had at least six months of follow-up and 36 (31\%) had at least one year of follow-up.

\section{Conclusions}

This preliminary clinical trial of patients undergoing soft tissue repair or reconstruction with a bioresorbable polymer appears to perform comparably to other commercially available devices. The lack of adverse events, mechanical failures, or infections further supports the safety of this device.

Review began 12/11/2020 Review ended 12/23/2020 Published 12/29/2020

\section{(๑) Copyright 2020}

Burnham et al. This is an open access article distributed under the terms of the Creative Commons Attribution License CC-BY 4.0., which permits unrestricted use, distribution, and reproduction in any medium, provided the original author and source are credited.
Categories: Orthopedics

Keywords: soft tissue fixation, lateral ankle ligament instability, deltoid ligament repair, bioresorbable implant, polymer implant, sonicanchor

\section{Introduction}

The quality of fixation of tendon or ligament to bone is a crucial consideration when selecting surgical anchors to perform soft tissue repair or reconstruction for musculoskeletal injuries [1]. Anchors have supplanted drill holes as an accepted method of surgically securing soft tissue to bone temporarily until definitive healing to bone occurs. Historically, initial anchors were composed of metal, but more recently they have evolved to radiolucent biopolymers [2-7]. The suture anchor has become an increasingly utilized method for suspensory fixation due to its ability to provide stronger fixation than punch-in anchors, as well as comparable strength and displacement to a knotted anchor [8-10].

Traditional anchors were made of metal. Imaging concerns coupled with technical complications prompted a shift to polymer-based implants [11-14]. While generally well accepted, polymer anchors occasionally fail due to material failure or loss of fixation caused by premature degradation [15-17]. A bioresorbable polymer composed of poly(L-lactide-co-D,L-lactide) was used to fabricate a biopolymer anchor with potential for enhanced fixation to cancellous bone (SonicAnchor ${ }^{\mathrm{TM}}$, Stryker, Kalamazoo, MI, USA). This soft tissue anchor utilizes controlled ultrasonic energy (SonicFusion ${ }^{\mathrm{TM}}$, Stryker) technology to "liquefy" the polymer anchor after an initial unicortical pilot hole has been drilled. The anchor then quickly reconstitutes within the cancellous bone allowing for enhanced interdigitation of the implant [18]. Recent biomechanical investigations have demonstrated enhanced strength of fixation to bone using this technology compared to popular, commercially available implants [19-21]. The goal of this preliminary trial was to collect and 


\section{Cureus}

analyze clinical follow-up data in a consecutive series of patients undergoing repair or reconstruction for soft tissue injuries in the foot and ankle using this biopolymer anchor.

\section{Materials And Methods}

Following Institutional Review Board approval, a retrospective chart review was performed on 116 consecutive patients undergoing soft tissue repair or reconstruction for injuries or disorders in the foot and ankle. A biopolymer, resorbable anchor (SonicAnchor) was used to secure fixation of soft tissue to bone. All patients who received at least one of these biopolymer, resorbable anchors were included in this study. There were no specific exclusion criteria for this study. Surgery was performed by two orthopedic foot and ankle surgeons in an academic medical center during a 3.5-year period from December 2015 to June 2019. Charts were abstracted for demographics and comorbidities, and surgery was performed. The success of surgery was defined as return to activities as tolerated with no restrictions or assistive devices. Adverse events included perioperative complications, infections, and failure to achieve the goals of the surgery.

\section{Results}

A total of 233 biopolymer resorbable implants (SonicAnchor) were implanted in 116 consecutive patients. There were 57 female and 59 male patients, with an average age of 42 years (range: $12-83$ years). The average BMI was 30.76 (standard deviation: 8.17 ) $\mathrm{kg} / \mathrm{m}^{2}$. Six patients were diabetic, 13 were current smokers, and 20 were previous smokers.

Table 1 demonstrates the primary diagnoses of patients who received at least one SonicAnchor bioresorbable suture anchor. Lateral ligamentous instability (58) and medial/deltoid ligamentous instability (49) were among the most common diagnoses. The operative procedures performed are listed in Table 2. The most common procedures were lateral ankle ligament reconstruction (38), deltoid ligament repair (33), and tendon repair (27).

\section{Primary Diagnoses}

Lateral ligamentous instability

Medial (deltoid) ligamentous instability

Chronic ankle instability

Foot drop

Syndesmotic injury

TABLE 1: Primary Diagnoses

\section{Operative Procedures}

Tendon repair

Tendon advancement

Achilles repair/lengthening

TABLE 2: Operative Procedures 


\section{Cureus}

Of the 116 patients, 108 (93.1\%) achieved clinical healing. Eight (6.9\%) patients were lost to follow-up prior to demonstrating clinical healing. All 108 patients who had adequate follow-up achieved clinical healing. Sixty-five (56\%) patients had at least six months of follow-up and 36 (31\%) had at least one year of followup (Table 3). Average follow-up duration was 309 days (range: 14-1,429 days). Two patients who followed up at their two-week visit for suture removal were lost to follow-up. The next shortest follow-up duration was at 41 days.

\begin{tabular}{|l|l|}
\hline Follow-Up & \\
\hline Patients with clinical healing & $108(93.1 \%)$ \\
\hline Lost to follow-up prior to clinical healing & $8(6.9 \%)$ \\
\hline Reoperation & $2(1.7 \%)$ \\
\hline Patients with six months of follow-up & $65(56 \%)$ \\
\hline Patients with one year of follow-up & $36(31 \%)$ \\
\hline Total patients & 116 \\
\hline
\end{tabular}

TABLE 3: Patient Follow-Up

Three patients required reoperation, with only one requiring revision secondary to persistent chronic instability. This patient was a 48 -year-old female with a history of ankle dislocation and subsequent development of chronic lateral ligament instability. She achieved clinical success following a second surgery consisting of lateral ankle ligament reconstruction with an allograft. Another reoperation patient was taken back to surgery as he sustained a bi-malleolar ankle fracture after falling from a tree. The patient demonstrated clinical healing by returning to full activities without restrictions and no pain at rest or with activity. This injury occurred eight months after his initial lateral ankle ligament reconstruction. The third patient had a history of foot drop for which she underwent a tibialis posterior tendon transfer. Her tendon transfer had stretched out over the course of 1.5 years and was prohibiting her from achieving neutral ankle dorsiflexion. She underwent an Achilles tendon lengthening and peroneus brevis and longus transfers to the dorsum of her midfoot.

Of the three reoperations, persistent chronic instability in one patient could be attributed to the failure of the biopolymer implant, but more likely due to the surgical technique. We believe the patient who sustained a bi-malleolar ankle fracture was a separate event that occurred after the biopolymer implant had already achieved clinical healing. The patient with a foot drop who underwent a tibialis posterior tendon transfer may have also been a result of implant failure. Although the tendon may have failed anywhere along its length, we could not exclude the possibility that the tendon may have failed to incorporate at the site of the implant. Of all the reoperations, none of the biopolymer implants was removed.

\section{Discussion}

Suture anchors have evolved as the preferred method of securing ligament or tendon to bone in reconstructive musculoskeletal surgery. Surgical anchoring has evolved from drill holes to metal suture anchors to the current preference for radiolucent biopolymer suture anchors. The bioresorbable polymer device used in this preliminary investigation (SonicAnchor) was created using a technology that can enhance the structural stability of the soft tissue anchor [21,22]. The goal was to create an anchor that could be "liquefied" and quickly reconstituted to achieve enhanced pullout strength and resistance to fatigue failure [19,23]. Figure 1 shows the short and simple steps for placing this bioresorbable anchor. This retrospective investigation suggests that the device appears to be safe and effective for use in orthopedic foot and ankle procedures in the short- and mid-term time frame. 


\section{Cureus}
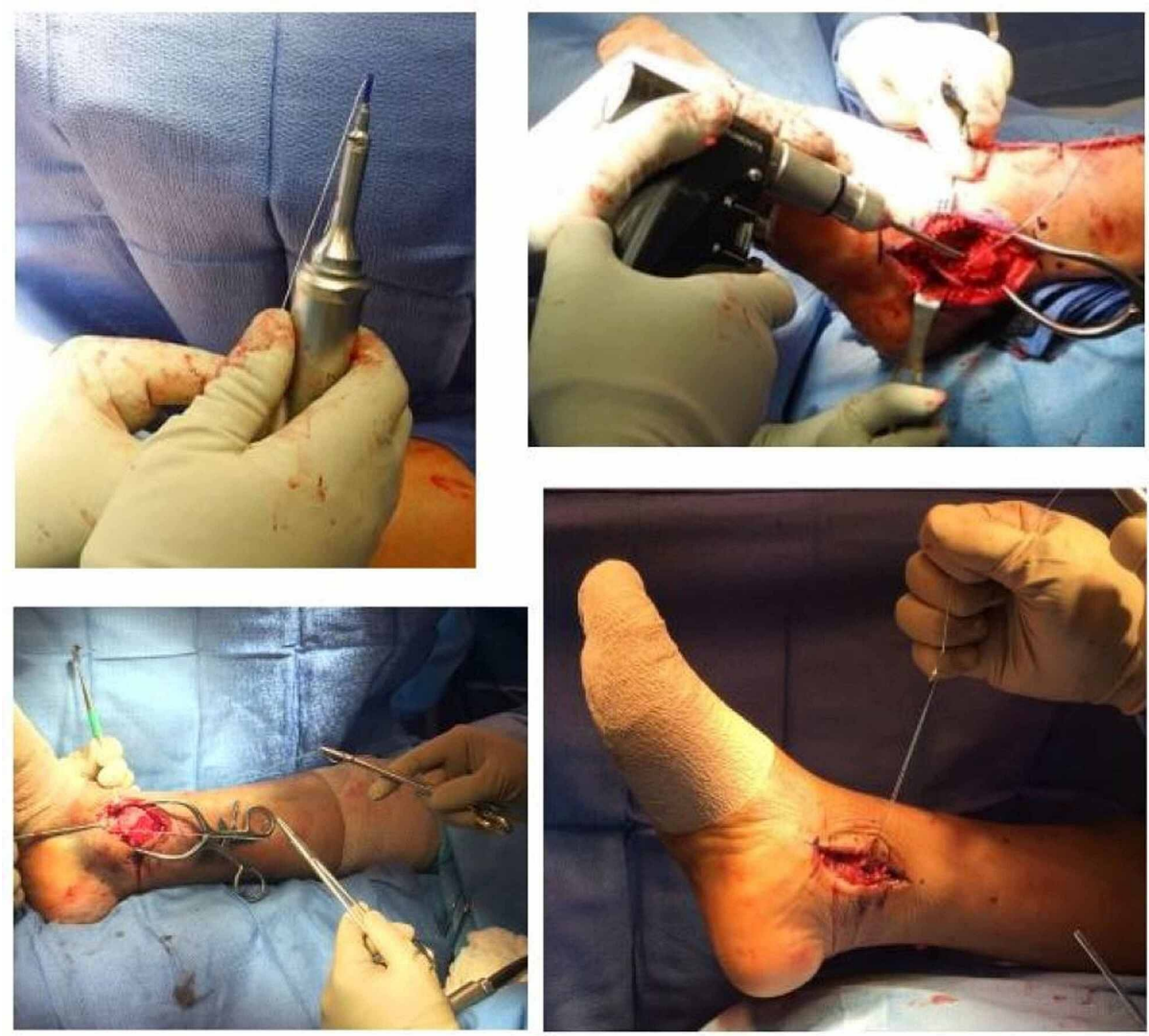

FIGURE 1: Clinical images of the bioresorbable polymer suture anchor. Top left: The insertion apparatus with a suture anchor attached at the tip of the device. Top right: Drilling of the desired suture anchor location. Bottom left: The suture anchor has been liquefied and has interdigitated within the cancellous bone. Two suture needles are held by needle drivers to be sewn into the desired tissue. Bottom right: The surgeon is picking up the weight of the lower extremity using the newly placed suture anchors, which demonstrates the strength of this suture anchor fixation.

One major drawback of using these novel bioresorbable polymer anchors is cost. The cost of each individual anchor varies based on the costs negotiated by individual hospitals. In contrast, the use of transosseous fixation in the form of bone tunnels requires no additional tools or implants. However, further studies need to be conducted to compare the rate of reoperation, clinical success, operative time, and cost of each method of fixation to truly evaluate the cost-effectiveness of each method.

The results of this retrospective case series compare favorably with similar ultrasonically interdigitated anchor use in a similar cohort of patients when examining the safety and efficacy profile [24,25].

The primary limitation to this preliminary clinical trial is the retrospective nature of this study. In addition, eight patients were lost to follow-up prior to demonstrating clinical healing. Two of the eight patients were lost to follow-up after their two-week postoperative visit. All patients were graduated from follow-up after they had demonstrated evidence of clinical healing, which limited the ability to evaluate patient status at long-term follow-up intervals. Follow-up for this study was highly influenced by surgeon preference to allow patients to follow-up on an as-needed basis after they had demonstrated clinical healing at their follow-up. Ideal future studies should include long-term follow-up and functional outcome scores.

\section{Conclusions}

This preliminary clinical trial of consecutive patients undergoing soft tissue repair or reconstruction with a novel bioresorbable suture anchor appeared to demonstrate a favorable safety and clinical effectiveness profile. The low incidence of adverse events (two of 116, 1.7\%), mechanical failures, or infections support the claims of this device. Further clinical studies need to be performed to support the perceived benefits of 


\section{Additional Information Disclosures}

Human subjects: Consent was obtained by all participants in this study. Loyola University Medical Center Institutional Review Board issued approval 210607. All required protocols by the LUMC IRB committee were followed and approval was granted in conjunction with conducting this study. Animal subjects: All authors have confirmed that this study did not involve animal subjects or tissue. Conflicts of interest: In compliance with the ICMJE uniform disclosure form, all authors declare the following: Payment/services info: All authors have declared that no financial support was received from any organization for the submitted work. Financial relationships: Adam P Schiff, Michael S Pinzur declare(s) personal fees from Stryker. Other relationships: All authors have declared that there are no other relationships or activities that could appear to have influenced the submitted work.

\section{References}

1. Cole BJ, Sayegh ET, Yanke AB, Chalmers PN, Frank RM: Fixation of soft tissue to bone: techniques and fundamentals. J Am Acad Orthop Surg. 2016, 24:83-95. 10.5435/JAAOS-D-14-00081

2. Ahmad CS, Gardner TR, Groh M, Arnouk J, Levine WN: Mechanical properties of soft tissue femoral fixation devices for anterior cruciate ligament reconstruction. Am J Sports Med. 2004, 32:635-40. $10.1177 / 0363546503261714$.

3. Latendresse K, Dona E, Scougall PJ, Shreuder FB, Puchert E, Walsh W: Cyclic testing of pullout sutures and micro-mitek suture anchors in flexor digitorum profundus tendon distal fixation. J Hand Surg Am. 2000, 30:471-8. 10.1016/j.jhsa.2004.10.014

4. Magen HE, Howell SM, Hull ML: Structural properties of six tibial fixation methods for anterior cruciate ligament soft tissue grafts. Am J Sports Med. 1999, 27:35-43. 10.1177/03635465990270011401

5. Milano G, Mulas PM, Zirano F, Piras S, Manunta A, Fabriciani C: Comparison between different femoral fixation devices for ACL reconstruction with doubled hamstring tendon graft: a biomechanical analysis. Arthroscopy. 2006, 22:660-8. 10.1016/j.arthro.2006.04.082

6. Park MC, Cadet ER, Levine WN, Bigliani LU, Ahmad CS: Tendon-to-bone pressure distributions at a repaired rotator cuff footprint using transosseous suture and suture anchor fixation techniques. Am J Sports Med. 2005, 33:1154-9. 10.1177/0363546504273053

7. Petre BM, Smith S, Jansson KS, et al.: Femoral cortical suspension devices for soft tissue anterior cruciate ligament reconstruction: a comparative biomechanical study. Am J Sports Med. 2013, 41:416-22. 10.1177/0363546512469875

8. Barber FA, Herbert MA: Cyclic loading biomechanical analysis of the pullout strengths of rotator cuff and glenoid anchors: 2013 update. Arthroscopy. 2013, 29:832-44. 10.1016/j.arthro.2013.01.028

9. Lo IKY, Burkhart SS, Athanaiou K: Cyclic loading biomechanical analysis of the pullout strengths of rotator cuff and glenoid anchors: 2013 update. Arthroscopy. 2004, 20:407-13. 10.1016/j.arthro.2004.01.022

10. Visscher LE, Jeffery C, Gilmour T, Anderson L, Couzens G: The history of suture anchors in orthopaedic surgery. Clin Biomech. 2019, 61:70-8. 10.1016/j.clinbiomech.2018.11.008

11. Barber FA, Herbert MA, Click JN: The ultimate strength of suture anchors . Arthroscopy. 1995, 11:21-8. 10.1016/0749-8063(95)90084-5

12. Kaar TK, Schenk RC, Wirth MA, Rockwood CA: Complications of metallic suture anchors in shoulder surgery: a report of 8 cases. Arthroscopy. 2001, 17:31-7. 10.1053/jars.2001.18246

13. Raeder BW, Figved W, Madsen JE, Frihagen F, Jacobsen SB, Andersen MR: Better outcome for suture button compared with single syndesmotic screw for syndesmosis injury: five-year results of a randomized controlled trial. Bone Joint J. 2020, 102:212-9. 10.1302/0301-620X.102B2.BJJ-2019-0692.R2.

14. Silver MD, Daigneault JP: Symptomatic interarticular migration of glenoid suture anchors . Arthroscopy. 2000, 16:102-5. 10.1016/s0749-8063(00)90136-1

15. Barber FA, Herbert MA, Coons DA, Boothby MH: Sutures and suture anchors-update 2006. Arthroscopy. 2006, 22:1063-9. 10.1016/j.arthro.2006.04.106

16. Klinger HM, Bucchorn GH, Heidrich G, Kahl E, Baums MH: Biomechanical evaluation of rotator cuff repairs in a sheep model: suture anchors using arthroscopic Mason-Allen stitches compared with transosseous sutures using traditional modified Mason-Allen stitches. Clin Biomech. 2008, 23:291-8. 10.1016/j.clinbiomech.2007.10.010

17. Meyer DC, Fucentese SF, Ruffieux K, Jacob HA, Gerber C: Mechanical testing of absorbable suture anchors . Arthroscopy. 2003, 19:188-93. 10.1053/jars.2003.50015

18. Sonic anchor system: operative technique guide. (2015). Accessed: October 7, 2020: http://az621074.vo.msecnd.net/syk-mobile-content-cdn/global-content-system/SYKGCSDOC-241248/1rvOVceLmUAHoabKrF4gm0Gi....

19. Adeosun SO, Lawal GI, Gbenebor OP: Characteristics of biodegradable implants. J Miner Mater Charact Eng. 2014, 2:88-106. 10.4236/jmmce.2014.22013

20. Boden SA, Boden AL, Mignemi D, Bariteau JT: Liquifying PLDLLA anchor fixation in Achilles reconstruction for insertional tendinopathy. Foot Ankle Spec. 2018, 11:162-7. 10.1177/1938640017740676

21. Meyer DC, Meyer J, Weber U, Mueller A, Koch PP, Gerber C: Ultrasonically implanted PLA suture anchors are stable in osteopenic bone. Clin Orthop Relat Res. 2005, 442:143-8. 10.1097/01.blo.0000185033.32220.57

22. Hembree WC, Tsai MA, Parks BG, Miller SD: Comparison of suture-based anchors and traditional bioabsorbable anchors in foot and ankle surgery. J Foot Ankle Surg. 2017, 56:3-7. 10.1053/j.jfas.2016.10.011

23. SonicAnchor ${ }^{\mathrm{TM}}$ : transforming the soft tissue experience. (2015). Accessed: October 7, 2020: http://az621074.vo.msecnd.net/syk-mobile-content-cdn/global-content-system/SYKGCSDOC-2- 


\section{Cureus}

43505/tPbFsPrTETpiN0QrJYkCXulC....

24. Kim ES, Lee KT, Park JS, Lee YK: Arthroscopic anterior talofibular ligament repair for chronic ankle instability with a suture anchor technique. Orthopedics. 2011, 34:819-25.

25. Warren B, Black N, Linscheid L, Panchbhavi V, Janney C: Use of ultrasonically interdigitated anchors in Haglund's deformity correction surgery [Online ahead of print]. Foot Ankle Spec. 2020,

10.1177/1938640020908455 